\title{
Atlas-Based Quantification of DTI Measures in a Typically Developing Pediatric Spinal Cord
}

\author{
(D) S. Shahrampour, (D) B. De Leener, (D) M. Alizadeh, (DD. Middleton, (D). Krisa, (D)A.E. Flanders, (D) S.H. Faro, (D). Cohen-Adad, and \\ (D) F.B. Mohamed \\ ow
}

\begin{abstract}
BACKGROUND AND PURPOSE: Multi-parametric MRI, provides a variety of biomarkers sensitive to white matter integrity, However, spinal cord MRI data in pediatrics is rare compared to adults. The purpose of this work was 3-fold: 1) to develop a processing pipeline for atlasbased generation of the typically developing pediatric spinal cord WM tracts, 2) to derive atlas-based normative values of the DTI indices for various WM pathways, and 3) to investigate age-related changes in the obtained normative DTI indices along the extracted tracts.
\end{abstract}

MATERIALS AND METHODS: DTI scans of 30 typically developing subjects (age range, 6-16 years) were acquired on a 3T MR imaging scanner. The data were registered to the PAM50 template in the Spinal Cord Toolbox. Next, the DTI indices for various WM regions were extracted at a single section centered at the C3 vertebral body in all the 30 subjects. Finally, an ANOVA test was performed to examine the effects of the following: 1) laterality, 2) functionality, and 3) age, with DTI-derived indices in 34 extracted WM regions.

RESULTS: A postprocessing pipeline was developed and validated to delineate pediatric spinal cord WM tracts. The results of ANOVA on fractional anisotropy values showed no effect for laterality $(P=.72)$ but an effect for functionality $(P<.001)$ when comparing the 30 primary WM labels. There was a significant $(P<.05)$ effect of age and maturity of the left spinothalamic tract on mean diffusivity, radial diffusivity, and axial diffusivity values.

CONCLUSIONS: The proposed automated pipeline in this study incorporates unique postprocessing steps followed by template registration and quantification of DTI metrics using atlas-based regions. This method eliminates the need for manual ROI analysis of WM tracts and, therefore, increases the accuracy and speed of the measurements.

ABBREVIATIONS: $\mathrm{AD}=$ axial diffusivity; $\mathrm{CV}=$ coefficient of variation; $\mathrm{DF}=$ dorsal funiculi; $\mathrm{FA}=$ fractional anisotropy; $\mathrm{LF}=$ lateral funiculi; $\mathrm{MD}=$ mean diffusivity; $\mathrm{RD}=$ radial diffusivity; $\mathrm{VF}=$ ventral funiculi

$\mathrm{n}$ recent years, DTI has shown promise as a noninvasive imaging biomarker for evaluating the tissue microstructure, ${ }^{1}$ which is sensitive to the integrity of the spinal cord WM. Recently, numerous studies have demonstrated the utility of DTI as a noninvasive tool to assess adult spinal cord WM microstructure. ${ }^{2,3}$ However, studies

Received February 9, 2021; accepted after revision April 19

From the Departments of Radiology (S.S., M.A., D.M., F.B.M.), Occupational Therapy (L.K.), and Radiology (A.E.F., S.H.F.), Thomas Jefferson University, Philadelphia, Pennsylvania; Department of Computer Engineering and Software Engineering (B.D.L.) and NeuroPoly Lab (J.C.-A.), Institute of Biomedical Engineering, Polytechnique Montreal, Montreal, Quebec, Canada; and Functional Neuroimaging Unit (J.C.-A.), Centre de Recherche de l'Institut Universitaire de Gériatrie de Montréal, Université de Montréal, Montreal, Quebec, Canada.

This work was supported by the National Institute of Neurological Disorders of the National Institutes of Health under award Nos. R01NS079635 and R01NS111113.

Please address correspondence to Feroze Mohamed, PhD, Jefferson Integrated MRI Center, Department of Radiology, Thomas Jefferson University, 901 Walnut St Philadelphia, PA 19107; e-mail: feroze.mohamed@jefferson.edu

- Indicates open access to non-subscribers at www.ajnr.org

Indicates article with online supplemental data.

http://dx.doi.org/10.3174/ajnr.A7221 related to the pediatric spinal cord have been limited so far. ${ }^{4,5}$ This issue highlights the importance of studying the pediatric spinal cord at a microstructural level and establishing normative values for various diffusion-derived indices obtained from the WM structures. A limited number of studies in pediatric subjects have demonstrated DTI indices differences between age and cord regions. ${ }^{4,5}$ However, all of these studies, so far, have used manual delineation of the WM structures in the pediatric spinal cord. ${ }^{5}$ Recently, methods have been developed that use automated extraction of the WM structures in the adult spinal cord. ${ }^{6}$ This approach alleviates any subjectivity in identification of WM structures. In this work, we have extended these methods to the pediatric spinal cord.

In summary, the aim of this work was 3-fold: 1) to develop a processing pipeline for atlas-based generation of pediatric spinal cord WM tracts. This provides a faster and more accurate method of tract delineation and eliminates the need for manual WM tract extraction. 2) to derive atlas-based normative values of the DTI parameters for various WM pathways at the C3 level of a typically developing pediatric spinal cord, and 3) to examine age-related changes in the derived normative DTI parameters along the extracted tracts. 


\section{MATERIALS AND METHODS Study Design and Population}

All subjects provided written informed consent obtained under a protocol approved by the institutional review board at Thomas Jefferson University. The study used 30 typically developing pediatric subjects (age range, 6-16 years; mean age, 12.38 [SD, 2.81] years). Following the recruitment, all subjects went through a brief assessment, and those with any abnormality of the nervous system were excluded.

\section{MR Imaging Protocol}

The scans were obtained using a $3 \mathrm{~T}$ Verio MR imaging scanner (Siemens) with a 4-channel neck and an 8-channel spine matrix coil. All the subjects underwent the scan protocol, consisting of an initial T2-weighted gradient-echo scout sagittal acquisition of the cervical and thoracic spinal cord. The sagittal scans were used to prescribe axial sections of both the cervical and thoracic spine. Imaging was then followed by obtaining an axial T2-weighted gradient recalledecho sequence, a sagittal T2-weighted 3D sampling perfection with application-optimized contrasts by using different flip angle evolution (3D-SPACE; Siemens) sequence, a sagittal TSE T1-weighted sequence, a sagittal TSE T2-weighted sequence, and axial diffusionweighted scans. We used the 3D-SPACE and diffusion-weighted data in this study. Diffusion-weighted images were acquired axially using 2 overlapping slabs to cover the entire cervical and thoracic spinal cord using an inner FOV spin-echo-based EPI pulse sequence with a tilted excitation plane. The inner FOV diffusion-weighted sequence consists of a spatially selective $2 \mathrm{D}$ radiofrequency excitation profile. ${ }^{7}$ This sequence has the advantage of reducing distortion artifacts in diffusion-weighted scans by enabling faster $\mathrm{k}$-space encoding via a reduction of phase-encoding steps. Manual shim and fat-saturation volume adjustments were also performed.

The imaging parameters of the diffusion-weighted acquisition for each slab are as follows: $\mathrm{FOV}=164 \mathrm{~mm}$, phase $\mathrm{FOV}=28.4 \%$ (47 mm), 3 averages of 20 diffusion directions, $6 b=0$ acquisitions, $b=800 \mathrm{~s} / \mathrm{mm}^{2}$, voxel size $=0.8 \times 0.8 \times 6 \mathrm{~mm}^{3}$, number of slices $=40, \mathrm{TR}=7900 \mathrm{~ms}, \mathrm{TE}=110 \mathrm{~ms}$, acquisition time $=8$ minutes and 49 seconds per slab. The T2-weighted SPACE imaging parameters used were: voxel size $=1 \times 1 \times 1 \mathrm{~mm}^{3}$, TR $=1500 \mathrm{~ms}$, TE $=$ $122 \mathrm{~ms}$, flip angle $=140^{\circ}$, number of averages $=2$, and acquisition time $=3$ minutes and 17 seconds (for each overlapping slab).

\section{Image Preprocessing}

The diffusion-weighted data from all the individual subjects were motion-corrected and later registered to the template (PAM50) in the Spinal Cord Toolbox (SCT; https://spinalcordtoolbox.com/ en/latest/) for performing atlas-based analysis of WM tracts. ${ }^{6}$ Registration is best done using the high-resolution anatomic image. In this study, all the pre- and postprocessing of the data was performed entirely within the framework of the SCT. ${ }^{8}$ These processes are described in detail below and illustrated in Fig 1.

\section{Procedure for Motion Correction}

All diffusion-weighted data were motion-corrected using a slice-wise motion-correction method. ${ }^{8}$ Specifically, using the sct_dmri_moco module from SCT, slice-by-slice planar translations within the diffusion-weighted data were estimated, while the regularization was constrained along the $\mathrm{z}$-direction (SliceReg algorithm).

\section{Procedure for Data Registration to the Template}

After applying motion correction on the diffusion-weighted data, we registered the data with the PAM $50{ }^{6}$ template available in the SCT. The registration procedure was done following a 2-step multimodal registration process within the SCT. The first step involved registration between the PAM50 template and an anatomical image of all the individual subjects. This was followed by a second registration between the template (in anatomical space) and the diffusion-weighted data of the same subjects. At the end of this process, the PAM50 atlas, which contains the various WM tracts, was registered to our own subject-specific diffusionweighted data. The following steps describe the registration process in detail:

1. Segmentation of spinal cord: The structural image and diffusion-weighted data of the cervical spine of all subjects were initially segmented using the sct_propseg ${ }^{9}$ module. The goal of this step was to extract a binary mask of the segmented spinal cord. Manual corrections were made on the images if the segmentation at certain locations was not satisfactory.

2. Labeling the anatomical image: In this step, the segmented structural images obtained from the previous step were labeled using the sct_label_vertebrae ${ }^{10}$ module. This step generated segmented cord labeled with vertebral levels.

3. Identifying vertebral levels along the cervical cord: Two vertebral levels (C3 and T1) were identified on the anatomical image using the sct_label_utils module. The main purpose of this step was to enable more accurate registration between the pediatric anatomical image and the PAM50 template by providing 2 reference voxels, one at the $\mathrm{C} 3$ level and a second one at the T1 level (orange arrows pointing to the corresponding intervertebral discs shown in Fig 1).

4. Next, each subject's individual T2-weighted image was registered to the PAM50 template using the sct_register_to_template module. This process includes straightening of the spinal cord and application of local deformations using a multistep nonlinear registration constrained in the axial plane and regularized in the $\mathrm{z}$-direction. A T2 template in the anatomic space (template2anat) and the corresponding warping field (warp_template2anat) were generated as the output of this step.

5. A second registration was then performed to register (template2anat); the image from the previous step to each subject's diffusion data using the sct_register_multimodal module. A template image in each individual's diffusion native space (template2diff) as well as the corresponding warping field (warp_anat2diff) were generated as the output of this step.

6. Concatenation of deformation fields: The warping fields from the last 2 steps of registrations were concatenated to create a single global transformation between the template and diffusion data (warp_template2diff).

7. Obtaining the WM atlas: Finally, the global warping field was used to warp the PAM50 template objects (ie, WM atlas) to the diffusion data. 


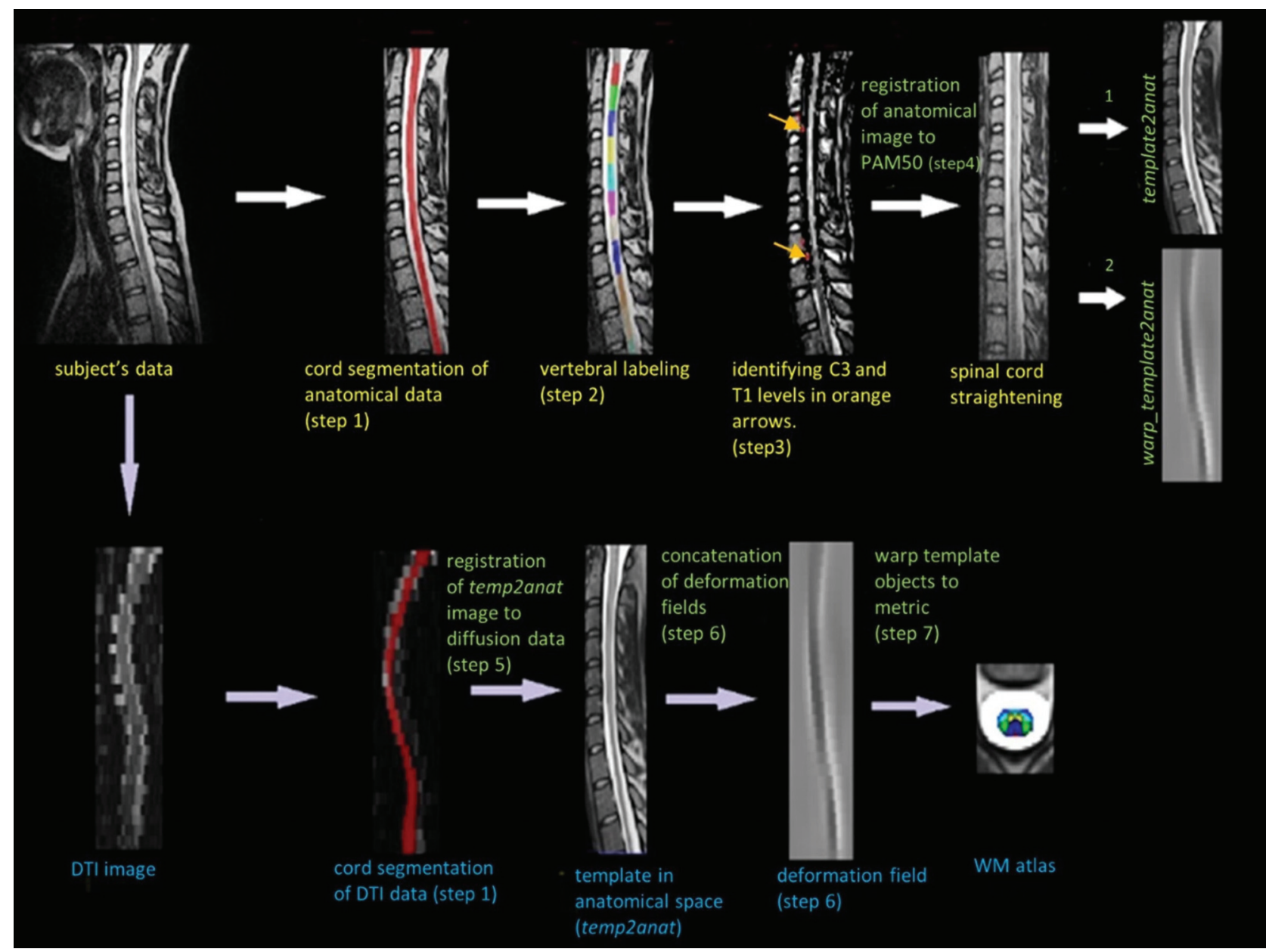

FIG 1. Overview of the template registration pipeline. Initially, T2-weighted scans are registered to the template (upper row). Diffusionweighted data acquired during the same scan session are then registered to the anatomic data, and PAM50 objects are warped to diffusion data (lower row) to generate the pediatric WM spinal cord atlas.

\section{Quantifying DTI Metrics Using the Atlas}

After successful registration was verified visually for each individual subject, DTI metrics were extracted (sct_extract_metric) for each subject at the C3 level using a weighted-average method. ${ }^{11}$ This step resulted in quantifying DTI metrics of fractional anisotropy (FA), mean diffusivity (MD), axial diffusivity (AD), and radial diffusivity $(\mathrm{RD})$ for 34 extracted WM labels from the PAM50 template. Of the 34 labels, 30 represent individual sensory and motor WM tracts, 3 represent combined labels of the dorsal funiculi (DF), lateral funiculi (LF), and ventral funiculi (VF), and 1 label was assigned to represent the entire WM itself. The normative values were obtained by averaging all these measurements across all 30 subjects. The full names of labels are provided in the Online Supplemental Data.

\section{Statistical Analysis}

GraphPad Prism software 8.0 (GraphPad Software), a DOS-based scientific graphing application, was used for statistical analysis of the data. For ease of visualization, DTI normative measurements of all the 34 labels were plotted using violin plots. One-way ANOVA was used to study the laterality (left/right), functionality (motor/sensory), and age-related changes among all the tracts. Linear regression analysis was used to compare age-related changes of DF, LF, and VF labels using DTI metrics. For the age-related analysis, the subject population was divided into 2 age groups: one group of younger children from 7-11 years of age and the older group from 12-16 years of age. The age groups were defined on the basis of a previous pediatric study. ${ }^{5}$ Throughout the study, a $P$ value of .05 was used to determine the statistical significance.

\section{RESULTS}

\section{Delineated WM Tracts}

Figure $2 A$ depicts a WM atlas of the human spinal cord. Figure $2 B$ illustrates a subset of tracts overlaid on a $b=0$ image at the $\mathrm{C} 3$ level in a representative subject from this study. The labeled WM tracts in this figure are explained in the figure legend. Figure $2 \mathrm{C}$ shows a sagittal T2-weighted cross-section of the spinal cord marked at the $\mathrm{C} 3$ level.

\section{Normative Values}

The developed automated WM pipeline was successful in extracting DTI values from all the various tracts across all 30 subjects 

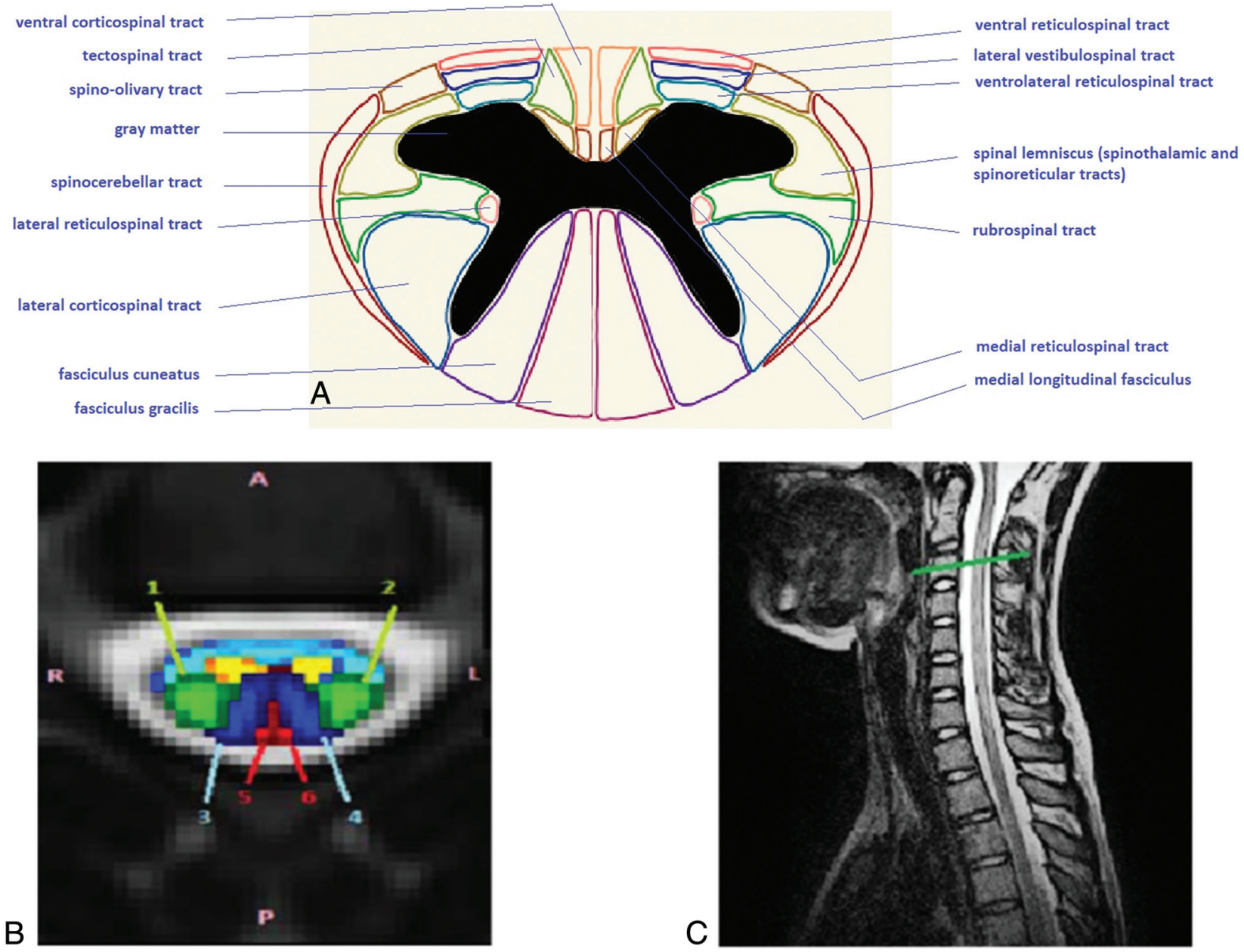

FIG 2. Spinal cord WM atlas. A, An atlas of spinal white matter tracts. $B$, Generated white matter atlas of the pediatric spinal cord overlaid on a $b_{0}$ image. The selected tracts are labeled with multiple colors: 1) right lateral corticospinal tract, 2) left lateral corticospinal tract, 3) right fasciculus cuneatus, 4) left fasciculus cuneatus, 5) right fasciculus gracilis, 6) left fasciculus gracilis, yellow: gray matter, light blue; unlabeled white matter. $C$, The C3 level is marked green on the sagittal T2-weighted scan.

(Fig 3). The violin plots were subdivided into the dorsal, lateral, and ventral funiculi as well as combined tracts (WM, DF, LF, and $\mathrm{VF}$ ). The FA in the dorsal column tracts ranged from 0.61 to 0.62 with the mean 0.62 (SD, 0.09). The corresponding values for lateral and ventral columns were 0.47 (SD, 0.12) and 0.28 (SD, 0.12 ), respectively.

$\mathrm{FA}, \mathrm{MD}$, and RD showed a higher level of dispersion in all the tracts, including dorsal, lateral, and ventral tracts compared with $\mathrm{AD}$. The coefficient of variation $(\mathrm{CV})$ of the measurements in FA ranged from $14.62 \%$ in the dorsal column to $45.74 \%$ in ventral tracts. Similar measurements in MD were observed $(23.75 \%$ to $34.06 \%$ from the dorsal-to-ventral tracts and in RD from $33.43 \%$ to $40.90 \%$, respectively). AD showed less variability across all the tracts, with a CV of $22 \%$ in the dorsal columns to $26 \%$ in the ventral tracts. As observed, ventral funiculi regions present larger variations compared with other WM regions. WM measurements from "combined tracts" had the lowest variation in FA, with a $\mathrm{CV}$ of $18.6 \%$, followed by the second lowest in $\mathrm{AD}(\mathrm{CV}=$ $20.11 \%)$. The VF had higher variation in all the metrics, with the average $\mathrm{CV}$ of $33 \%$ compared with its counterparts DF and LF $\left(\mathrm{CV}_{(\mathrm{DF})}=22 \%, \mathrm{CV}\left({ }_{\mathrm{LF}}\right)=25 \%\right)$.
The Table compares the diffusion indices obtained for 6 selected tracts shown in Fig $2 B$. From all the tracts in the Table, the left fasciculus gracilis shows the highest FA of 0.66 averaged among all 30 subjects with the lowest variation (CV $=14.02 \%)$. FA was the lowest in the right lateral corticospinal tract with a higher CV of $18.26 \%$.

\section{Effects of Laterality, Functionality, and Age}

Results of ANOVA using FA, MD, RD, and AD showed no significant difference $(P>.05)$ between a total of 15 left and 15 right tracts. However, when tested for functionality (i.e., whether a tract is sensory or motor), FA and MD showed a significant difference $\left(P_{\mathrm{FA}}=3.7 \mathrm{E}^{-5}, P_{\mathrm{MD}}=.043\right)$, but $\mathrm{AD}$ and $\mathrm{RD}$ showed no effect.

Examining the effect of age with each single individual tract as well as combined tracts revealed significant differences among the maturation of some tracts and age. Among all 34 selected tracts studied, only the left spinal lemniscus (spinothalamic and spinoreticular tracts) showed an effect with age using $\mathrm{MD}, \mathrm{RD}$, and $\operatorname{AD}(P<.05)$. Additionally, linear regression analysis showed a positive correlation between FA and age as shown in Fig $4 A$, while $\mathrm{MD}, \mathrm{RD}$, and $\mathrm{AD}$ (Fig $4 B-D$ ) showed a negative correlation 

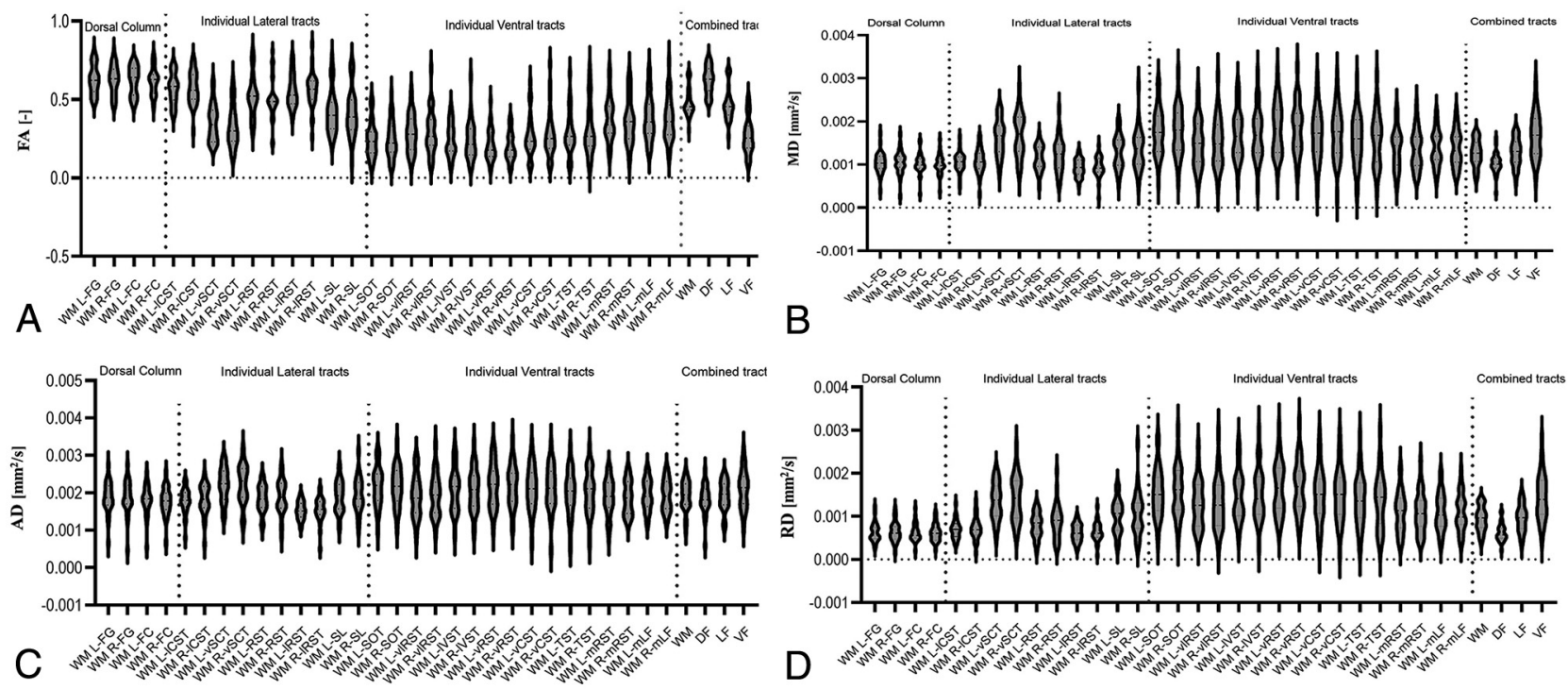

FIG 3. Violin plots of normative $F A(A), M D(B), A D(C)$, and RD $(D)$ indices averaged across 30 healthy pediatric subjects for 34 ROIs. ROls are subdivided in to 30 WM tracts; 3 combined labels of DF, LF, and VF; and 1 label for the entire WM. IVST indicates lateral vestibulospinal tract; VRST, ventral reticulospinal tract; FC, fasciculus cuneatus; ICST, lateral corticospinal tract; TST, tectospinal tract; SL, spinal lemniscus (spinothalamic and spinoreticular tracts); FG, fasciculus gracilis; SOT, spino-olivary tract; mLF, medial longitudinal tract; vCST, ventral corticospinal tract; vIRST, ventrolateral reticulospinal tract; IRST, lateral reticulospinal tract; RST, rubrospinal tract; $v S C T$, ventral spinocerebellar tract; mRST, medial reticulospinal tract; WM, white matter; DF, dorsal column; LF, lateral funiculi; VF, ventral funiculi; L, left; R, right.

Results of DTI normative values for 6 selected tracts illustrated in Fig 2B

\begin{tabular}{|c|c|c|c|c|c|}
\hline Group & WM Tracts & FA (Average) (SD) & MD (Average) (SD) & RD (Average) (SD) & AD (Average) (SD) \\
\hline 1 & Right lateral corticospinal tract & $0.56(S D, 0.09)$ & $1.27(S D, 0.20)$ & $0.87(S D, 0.24)$ & $2.09(\mathrm{SD}, 0.27)$ \\
\hline 2 & Left lateral corticospinal tract & $0.60(S D, 0.08)$ & $1.17(\mathrm{SD}, 0.14)$ & $0.78(S D, 0.18)$ & $1.95(\mathrm{SD}, 0.24)$ \\
\hline 3 & Right fasciculus cuneatus & $0.64(S D, 0.08)$ & 1.14 (SD, 0.18) & 0.72 (SD, 0.18) & $2.00(\mathrm{SD}, 0.31)$ \\
\hline 4 & Left fasciculus cuneatus & $0.66(S D, 0.08)$ & 1.13 (SD, 0.15) & $0.68(S D, 0.18)$ & $2.05(S D, 0.26)$ \\
\hline 5 & Right fasciculus gracilis & 0.65 (SD, 0.06) & 1.20 (SD, 0.19) & $0.72(S D, 0.20)$ & $2.16(S D, 0.33)$ \\
\hline 6 & Left fasciculus gracilis & $0.66(S D, 0.07)$ & $1.20(S D, 0.19)$ & $0.72(S D, 0.20)$ & $2.15(\mathrm{SD}, 0.31)$ \\
\hline
\end{tabular}

in all 3 combined tracts (dorsal, ventral, and lateral funiculi). MD showed significant differences with age in the VF and DF $(P<.05)$, whereas AD showed an effect in the LF and VF but not the DF. Similar analysis showed no effect for FA but a significant effect for $\mathrm{RD}$ in all 3 combined tracts of $\mathrm{DF}, \mathrm{LF}$, and $\mathrm{VF}(P<.05)$.

\section{DISCUSSION}

In this study, we developed a processing pipeline for automatic generation of pediatric spinal cord WM tracts. The acquisition was followed by processing the data with a unique pipeline and registration to an external template. The quantification of DTI indices was facilitated with the atlas incorporated in PAM50, ${ }^{6}$ followed by establishing DTI normative values for 34 WM pathways. Former studies have addressed the generation of the spinal cord atlas in the adult population. ${ }^{8,11}$ However, no atlas of WM pathways in the pediatric spinal cord is currently available. This study is the first to propose a pipeline for automated WM tract extraction in pediatrics enabling atlas-based analysis of the spinal cord.

\section{WM Tract-generation Pipeline}

The WM atlas of the spinal cord based on DTI enables automated atlas-based analysis of WM pathways. The extracted tracts in this study are aligned with the PAM50 T2-weighted template, which minimizes the interpolation errors during diffusion metrics extraction.

In the SCT, a digitized version of the Gray's Anatomy spinal cord WM atlas is merged with PAM50. Currently, this atlas is the most detailed atlas of human anatomy. ${ }^{11,12}$ However, one of the limitations of this atlas is that it does not take into account the between-subject variability of the spinal cord shape, which has been addressed in previous studies. ${ }^{13}$ We incorporated a multistep registration method to register the pediatric diffusion-weighted data to the adult PAM50 template. To make this process more feasible, we used the sct_label_utils tool from the SCT to create 2 landmarks at the $\mathrm{C} 3$ and $\mathrm{T} 1$ vertebral levels in the anatomical image of each subject. This method considers the height difference between the adult and pediatric population and adjusts for the discrepancies. We then used a BSplineSyN ${ }^{14}$ algorithm in the final step of the multimodal registration to preserve the shape of the cord. We visually examined the outcome of the registration in each individual subject, and the results were satisfactory. The suggested method provides a fully automated and accurate WM segmentation compared with wholecord manual ROI drawings used in similar spinal cord DTI studies such as the work of Reynolds et al. ${ }^{4}$ The manual ROI drawing performed in the studies of Reynolds et $\mathrm{al}^{4}$ and Saksena et $\mathrm{al}^{5}$ is also prone to partial volume contamination, which can be alleviated with our proposed automated pipeline. ${ }^{15}$ 
Ventral Funiculi (VF)

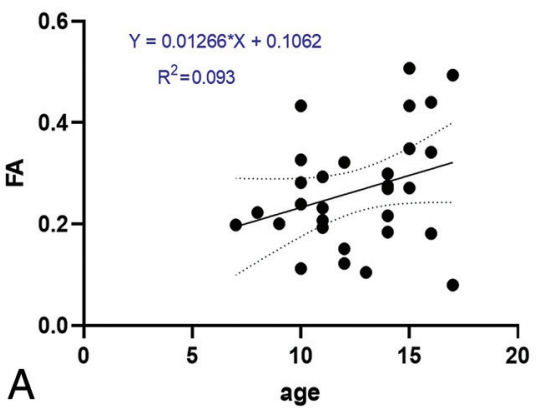

Ventral Funiculi (VF)

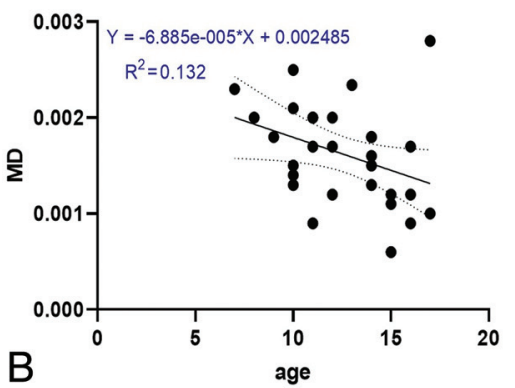

Ventral Funiculi (VF)

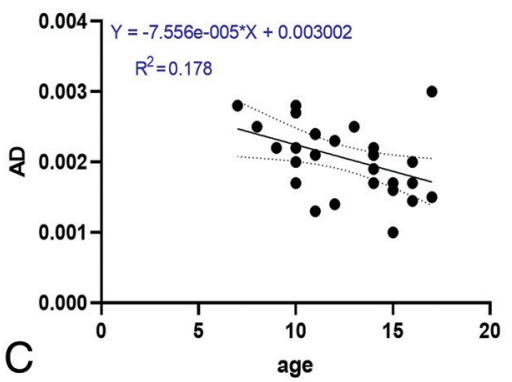

Ventral Funiculi (VF)

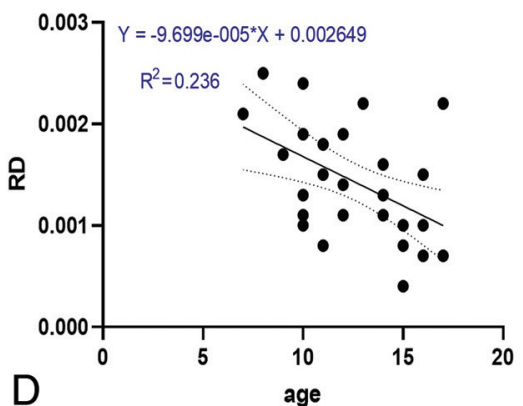

Dorsal Funiculi (DF)

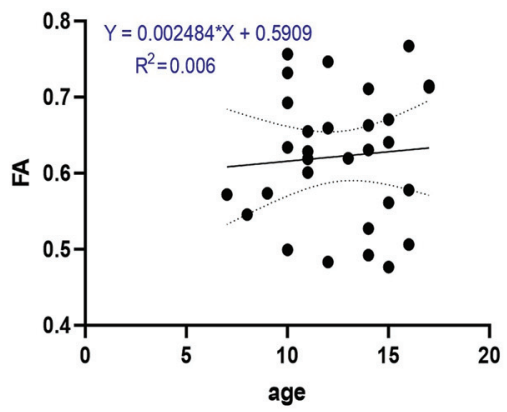

Dorsal Funiculi (DF)

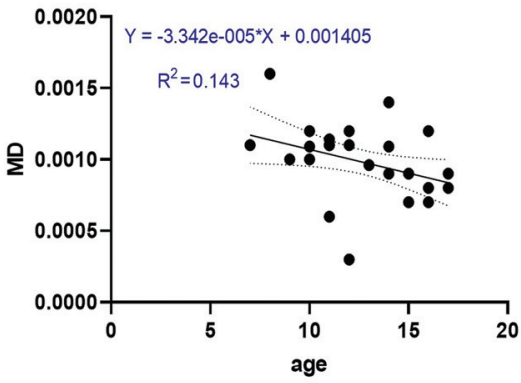

Dorsal Funiculi (DF)

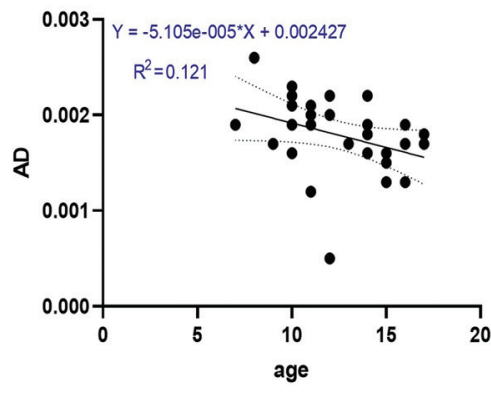

Dorsal Funiculi (DF)

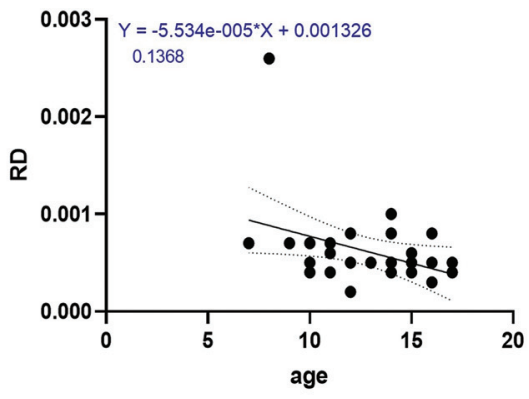

Lateral Funiculi (LF)

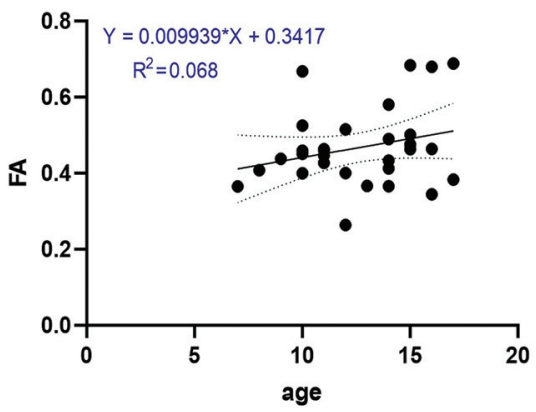

Lateral Funiculi (LF)
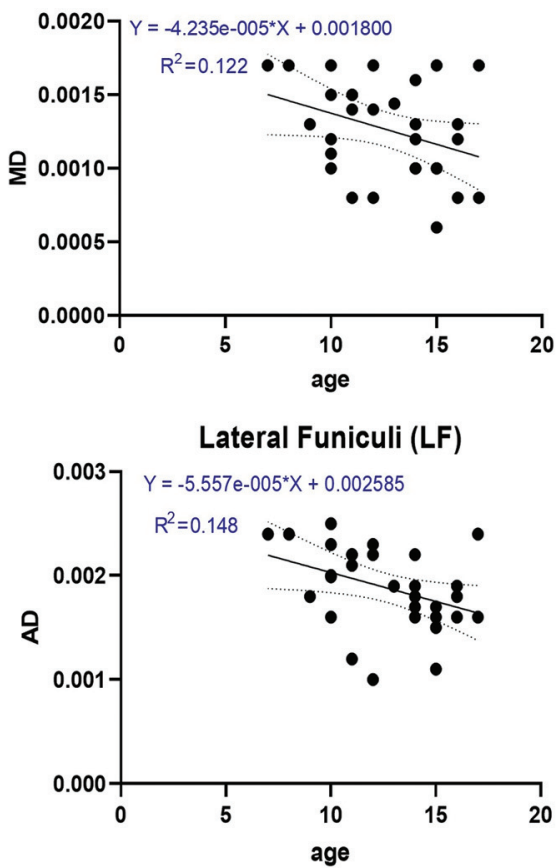

Lateral Funiculi (LF)

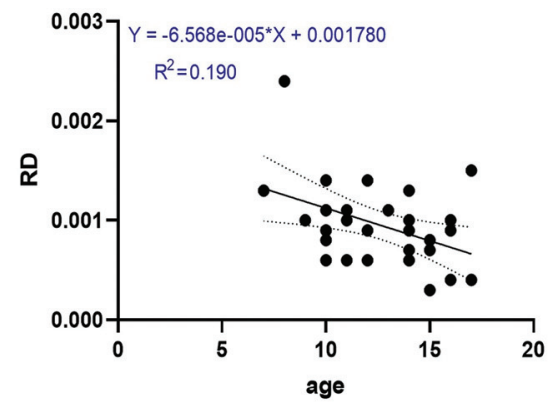

FIG 4. Linear regression plots showing the relationship between age and FA $(A), M D(B), A D(C)$, and RD $(D)$ in the ventral, lateral, and dorsal funiculi. Solid lines indicate the age-related trend, and dashed lines indicate $95 \%$ confidence intervals.

\section{DTI Metrics Quantification of the Spinal Cord}

In this work, we obtained normative values of diffusion data at the C3 vertebral level for ventral, dorsal, and lateral spinal cord funiculi as well as a specific subset of WM tracts in the healthy pediatric population. We believe that these values could be useful when comparing healthy and patient populations. ${ }^{16}$

Considering the various known spinal cord complications, study of spinal cord WM and characterizing its microstructure noninvasively will help in accurate understanding and diagnosis of disease and pathologies.

Injury to the spinal cord due to traumatic or nontraumatic events and subsequently to white matter tracts in general leads to specific dysfunction and various clinical disabilities. Therefore, identifying the various specialized types of the WM pathways in the cord that may have been damaged is crucial in helping the prognosis of functional recovery and disease management. 
Note that our analysis focused only on a single spinal cord level (C3 vertebral level) due to the better performance of proposed segmentation and registration algorithms with lower interrater variability reported by Prados et al. ${ }^{17}$ Also, as shown by Losseff et al, ${ }^{18} \mathrm{C} 2 / \mathrm{C} 3$ level results are the most reproducible values when studying spinal cord atrophy. In addition, the work of Stevenson et $\mathrm{al}^{19}$ suggests that the measurement variability that occurs using other spinal levels could be eliminated by focusing the analysis on the $\mathrm{C} 2 / \mathrm{C} 3$ intervertebral disc. This possibility is due to the excellent contrast of the cord and CSF at the C3 level (i.e., a capacious CSF pool).

When we investigated the function of age and DTI indices, our observed age-related trend of DTI indices matches that of other spinal cord studies. ${ }^{5}$ However, the lack of a statistical significance effect with a decrease in $\mathrm{AD}$ observed in the dorsal columns suggests that the proportion of larger-sized axons increases with the child's growth. ${ }^{4}$

In comparing the DTI metrics of the funiculi with age, we found no significant difference between FA values of the LF, DF, and VF. However, MD, RD, and $\mathrm{AD}$ values of the VF showed a significant effect with age-related changes. These differences could reflect the variability of the axon diameter among these funicles ${ }^{20}$ containing different kinds of tracts. Despite the lack of a significant effect observed between the FA and age, the correlation of age with FA specifically and other DTI-derived indices generally was consistent with that in former studies. ${ }^{5,21}$

While examining the effect of age on each individual tract in this study, we observed that there is a relationship of diffusivity metrics and maturation of some tracts (i.e., the spinothalamic tract). This could be because of the myelination process in different tracts. Myelination constitutes the last stage in the development of WM, which begins in utero in the second trimester of pregnancy and continues up to the end of adolescence. ${ }^{22}$ There are several factors that affect the rate of myelination, including the type of fiber, where faster rates are observed in sensory compared with motor tracts. ${ }^{23}$ We believe that this could justify the significant effect of spinothalamic tract (a somatosensory tract) maturation in DTI indices in our study.

We also showed that there is no significant difference in the laterality (left/right) of the tracts. These findings suggest structural similarity between the left- and right-sided tracts. In a study by Duval et $\mathrm{al}^{24}$ the authors have shown similar average axon diameters in identical tracts from the left and right sides of the spinal cord. Although there was no significant difference in the laterality of the tracts, ANOVA showed a significant difference between sensory (i.e., fasciculus gracilis, fasciculus cuneatus) and motor (i.e., corticospinal tract) pathways. We believe that this finding is related to the microstructural organization of the sensory and motor WM tracts. The anatomic variation of axon diameter is noticeable; whereas the dorsal column fasciculus gracilis contains mainly small-diameter axons, the corticospinal tract in the lateral and ventral columns contains a combination of large- and small-diameter axons. ${ }^{25}$ Another anatomic variation to consider is the fiber density variation along the entire length of the spinal cord, especially at the exit points of the brachial and lumbosacral plexuses. ${ }^{12}$ In a study by Ohnishi et al, ${ }^{26}$ the authors reported a decrease in the number of myelinated fibers of the fasciculus gracilis in humans between C3 and T5. Terao et $\mathrm{al}^{27}$ also reported an association of axonal diameter variation at the vertebral level in the human lateral corticospinal tract. These observations are in accordance with previous histology work ${ }^{28}$ in humans,' emphasizing the importance of broader studies of histologic validation in the ex vivo human spinal cord.

\section{Limitations}

Due to the diffusion data, SNR limitations, lack of anatomical landmarks, and the smaller size of the cord in children, there could be higher intrasubject variability resulting from spatial misalignment during registration of diffusion data to structural scans. This limitation emphasizes the need for tools that provide robust preprocessing, normalization, and motion correction for spinal cord diffusion data. Another limitation stems from the intrinsic variability of spinal cord diffusion imaging, which induces inaccuracies in metrics quantification. This issue, along with the selected registration algorithms method of metric extraction and partial volume effects, contributes to intersubject variability, particularly with a smaller cord size. An additional limitation is the small number of subjects as well as the narrow age range of our population as opposed to other pediatric diffusion studies. ${ }^{4}$ All of the aforementioned limitations could be contributing factors to observed discrepancies in our results compared with other similar studies.

\section{CONCLUSIONS}

We demonstrated the feasibility of a fully automated WM tract extraction in the pediatric spinal cord. We also established normative values for DTI-derived indices, looking at specific WM regions of the cord. These results show that the presented values are microstructure-sensitive and evolve in the pediatric spinal cord during development. This finding warrants future work with spinal cord patients (i.e., spinal cord injury) as well as an examination of DTI-derived metrics in the entire cord compared with a single $\mathrm{C} 3$ section.

Disclosures: Laura Krisa-RELATED: Grant: National Institutes of Health.* *Money paid to the institution.

\section{REFERENCES}

1. Basser PJ. Inferring microstructural features and the physiological state of tissues from diffusion-weighted images. NMR Biomed 1995;8:333-44 CrossRef Medline

2. Rossi C, Boss A, Steidle G, et al. Water diffusion anisotropy in white and gray matter of the human spinal cord. J Magn Reson Imaging 2008;27:476-82 CrossRef Medline

3. Wheeler-Kingshott CA, Hickman SJ, Parker GJ, et al. Investigating cervical spinal cord structure using axial diffusion tensor imaging. Neuroimage 2002;16:93-102 CrossRef Medline

4. Reynolds BB, By S, Weinberg QR, et al. Quantification of DTI in the pediatric spinal cord: application to clinical evaluation in a healthy patient population. AJNR Am J Neuroradiol 2019;40:1236-41 CrossRef Medline

5. Saksena S, Middleton DM, Krisa L, et al. Diffusion tensor imaging of the normal cervical and thoracic pediatric spinal cord. AJNR Am J Neuroradiol 2016;37:2150-57 CrossRef Medline

6. De Leener B, Fonov VS, Collins DL, et al. PAM50: unbiased multimodal template of the brainstem and spinal cord aligned with the ICBM152 space. Neuroimage 2018;165:170-79 CrossRef Medline 
7. Finsterbusch J. Improving the performance of diffusion-weighted inner field-of-view echo-planar imaging based on 2D-selective radiofrequency excitations by tilting the excitation plane. J Magn Reson Imaging 2012;35:984-92 CrossRef Medline

8. De Leener B, Lévy S, Dupont SM, et al. SCT: Spinal Cord Toolbox, an open-source software for processing spinal cord MRI data. Neuroimage 2017;145:24-43 CrossRef Medline

9. De Leener B, Kadoury S, Cohen-Adad J. Robust, accurate and fast automatic segmentation of the spinal cord. Neuroimage 2014;98:52836 CrossRef Medline

10. Ullmann E, Pelletier Paquette JF, Thong WE, et al. Automatic labeling of vertebral levels using a robust template-based approach. Int J Biomed Imaging 2014;2014:719520 CrossRef Medline

11. Lévy S, Benhamou M, Naaman $C$, et al. White matter atlas of the human spinal cord with estimation of partial volume effect. Neuroimage 2015;119:262-71 CrossRef Medline

12. Standring S. Gray's Anatomy: The Anatomical Basis of Clinical Practice. 40th ed. Elsevier Churchill Livingstone; 2008

13. Fradet L, Arnoux PJ, Ranjeva JP, et al. Morphometrics of the entire human spinal cord and spinal canal measured from in vivo highresolution anatomical magnetic resonance imaging. Spine (Phila Pa 1976) 2014;39:E262-69 CrossRef Medline

14. Tustison NJ, Avants BB. Explicit B-spline regularization in diffeomorphic image registration. Front Neuroinform 2013;7:39 CrossRef Medline

15. Cohen-Adad J, Descoteaux M, Rossignol S, et al. Detection of multiple pathways in the spinal cord using q-ball imaging. Neuroimage 2008;42:739-49 CrossRef Medline

16. By S, Xu J, Box BA, et al. Application and evaluation of NODDI in the cervical spinal cord of multiple sclerosis patients. Neuroimage Clin 2017;15:333-42 CrossRef Medline

17. Prados F, Cardoso MJ, Yiannakas MC, et al. Fully automated grey and white matter spinal cord segmentation. Sci Rep 2016;6:36151 CrossRef Medline
18. Losseff NA, Webb SL, O'Riordan JI, et al. Spinal cord atrophy and disability in multiple sclerosis. a new reproducible and sensitive MRI method with potential to monitor disease progression. Brain 1996;119(Pt 3):701-08 CrossRef Medline

19. Stevenson VL, Leary SM, Losseff NA, et al. Spinal cord atrophy and disability in MS: a longitudinal study. Neurology 1998;51:234-38 CrossRef Medline

20. Assaf Y, Blumenfeld-Katzir T, Yovel Y, et al. AxCaliber: a method for measuring axon diameter distribution from diffusion MRI. Magn Reson Med 2008;59:1347-54 CrossRef Medline

21. Alizadeh M, Fisher J, Saksena S, et al. Age-related diffusion and tractography changes in typically developing pediatric cervical and thoracic spinal cord. Neuroimage Clin 2018;18:784-92 CrossRef Medline

22. Brody BA, Kinney HC, Kloman AS, et al. Sequence of central nervous system myelination in human infancy, I: an autopsy study of myelination. J Neuropathol Exp Neurol 1987;46:283-301 CrossRef Medline

23. Coll G, de Schlichting E, Sakka L, et al. Assessment of maturational changes in white matter anisotropy and volume in children: a DTI study. AJNR Am J Neuroradiol 2020;41:1726-32 CrossRef Medline

24. Duval T, McNab JA, Setsompop K, et al. In vivo mapping of human

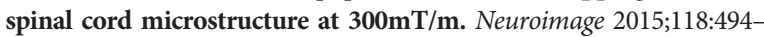
507 CrossRef Medline

25. Saliani A, Perraud B, Duval T, et al. Axon and myelin morphology in animal and human spinal cord. Front Neuroanat 2017;11:129 CrossRef Medline

26. Ohnishi A, O'Brien PC, Okazaki H, et al. Morphometry of myelinated fibers of fasciculus gracilis of man. J Neurol Sci 1976;27:163-72 CrossRef Medline

27. Terao S, Sobue G, Hashizume Y, et al. Age-related changes of the myelinated fibers in the human corticospinal tract: a quantitative analysis. Acta Neuropathol 1994;88:137-42 CrossRef Medline

28. Nieuwenhuys R, Voogd J, van Huijzen C. The Human Central Nervous System: A Synopsis and Atlas. 4th ed. Springer; 2007 